\title{
Classification of radar images with different methods of image preprocessing
}

\author{
A A Borodinov ${ }^{1}$ and V V Myasnikov ${ }^{1,2}$ \\ ${ }^{1}$ Samara National Research University, Moskovskoye shosse34, Samara, Russia, 443086 \\ ${ }^{2}$ Image Processing Systems Institute - Branch of the Federal Scientific Research Centre \\ "Crystallography and Photonics" of Russian Academy of Sciences, Molodogvardeyskaya str. \\ 151, Samara, Russia, 443001
}

\begin{abstract}
This work is aimed at comparing the classification algorithms and methods of machine learning with various methods of preliminary processing of radar images. Preprocessing step includes speckle noise filtering and object orientation normalization on the image. In comparison, the following classification algorithms were considered: Decision Tree, Support Vector Machine, K Nearest-Neighbor method, Random Forest, AdaBoost where decision tree was used as a weak classifier, Convolutional Neural Network and Residual Neural Network. The principal component analysis was applied to reduce the dimension. The study was carried out on the objects from the base of radar images MSTAR.
\end{abstract}

\section{Introduction}

Radar satellite imagery obtained with synthetic aperture radar received its distribution due to independence from weather and natural illumination. The recognition of objects on radar images is used in various fields, such as agriculture, forestry, geology, ecology, and also in the work of the emergency and rescue services. Synthetic aperture radar is usually an air or space radar system that uses the flight path of a platform to simulate an extremely large antenna, which makes it possible to create images of remote sensing of the earth with high resolution.

A certain complexity in the processing of the images obtained is the speckle noise that is present on the radar images. To improve the accuracy of the classification of images, the orientation of the object is also normalized. In most cases, the angle of rotation of the target is unknown. In this paper, we consider three methods commonly used in scientific articles to find the angle of rotation: a method that relies on the use of image moments; a method based on the Hough Transform, in which there is a search for long straight lines in the image, which are taken beyond the boundaries of the object; a method based on finding a rectangle with a minimum area describing the object.

The paper compares classical algorithms for the classification of radar images for various methods of their preliminary processing and methods based on the use of convolutional neural networks [8]. All the experiments were carried out using the generally available database of radar images of MSTAR military equipment [1].

The paper is organized as follows. In the second section, the statement of the classification problem is given. The third paragraph describes methods of preliminary image processing and compares the accuracy of finding the angle of rotation. In the fourth chapter, a method for reducing the 
dimensionality of the characteristic space is described. In the fifth chapter, the classification algorithms under investigation are described. The sixth chapter presents the results of experimental studies. The conclusion contains conclusions and plans for further research. In the end of the work, thanks and a list of used literature are indicated.

\section{Statement of the classification problem}

The task of object recognition on an image can be divided into two main subtasks:

- search for an object in the image and selection of areas of interest;

- recognition and classification of the found object or area of interest. [1]

The first subtask is aimed at finding objects for classification. Often information about the location, size, orientation, availability, and a number of goals is initially missing. In this case, it is necessary to determine the unknown parameters required for further selection of the object or local area of interest.

The second subtask is applied to the entire image and allows you to decide which of the several classes the image being processed belongs to. The decision function for each feature vector relates the object to the corresponding class. In this paper, we consider only the classification problem.

Due to the need to process a large number of images for training and testing, as well as a low performance of some algorithms, there is a need to reduce the dimensionality of the feature space. There are various methods used to solve this problem. These methods include the most popular methods: the principal component analysis, factor analysis, the independent component analysis, Selforganizing map and others. In article [10] the authors use a convolutional neural network to reduce the dimension and compare the results with SVM [11]. In this paper, the principal component analysis is applied.

\section{Pre-processing images}

The preliminary processing of images consists of two stages. At the first stage, speckle noise is filtered by the Lee filter and threshold processing. At the second stage, one of the methods of normalizing the orientation of the object on the image is applied.

The method based on the moments of the image allows us to obtain the required rotation angle using the following formula, where

$$
\begin{aligned}
& \mu_{11}^{\prime}=\mu_{11} / \mu_{00}=M_{11} / M_{00}-x y, \\
& \mu_{20}^{\prime}=\mu_{20} / \mu_{00}=M_{20} / M_{00}-x^{2}, \\
& \mu_{02}^{\prime}=\mu_{02} / \mu_{00}=M_{02} / M_{00}-y^{2}:
\end{aligned}
$$

$$
\varphi=\frac{1}{2} \operatorname{arctg} \frac{2 \mu_{11}^{\prime}}{\mu_{20}^{\prime}+\mu_{02}^{\prime}}+v \frac{\pi}{2}(v=0,1, \ldots)
$$

In the method based on finding the minimum area of the rectangle describing the object in the image, the rotation angle is calculated as the angle between the horizontal and one of the sides of the rectangle. It is assumed that the rectangle will describe the object on the image in such a way that the longest side of the object will be described by the long side of the rectangle. To isolate the boundaries of an object, the Canny algorithm is used. The objects from the MSTAR database, described by the minimum area rectangle are shown in Figure 1.
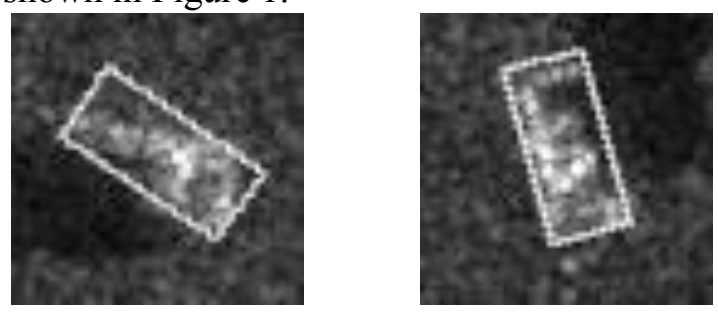

Figure 1. On the left is an example of an incorrect finding of a rectangle, the right selection of an object is shown on the right.

Figure 2 shows the scheme of the preprocessing algorithm. 


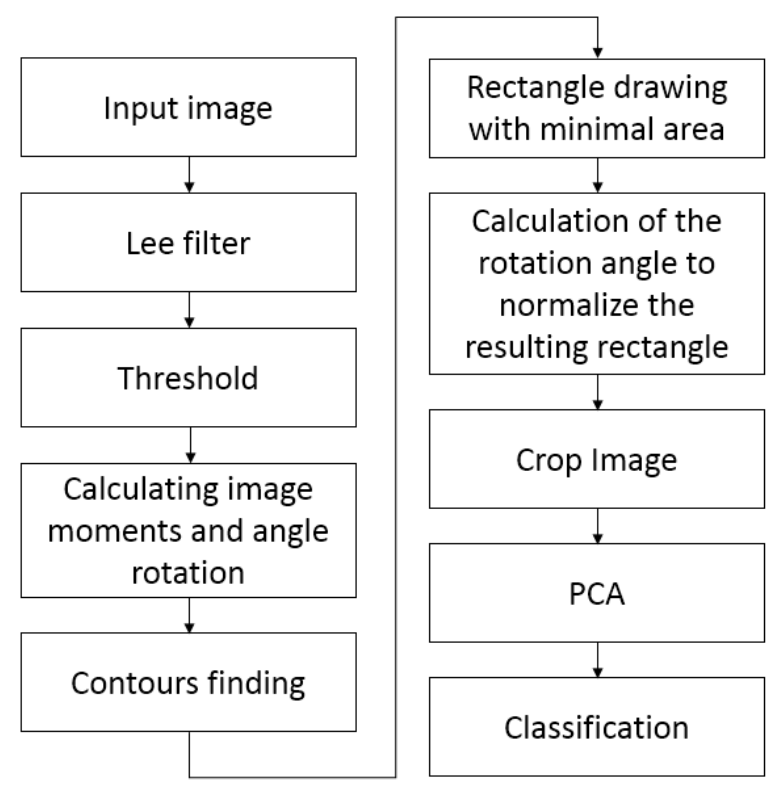

Figure 2. Preprocessing algorithm using a method based on finding the rectangle of the minimum area.

The method based on the Hough Transform involves finding long straight lines on the image that can be the boundaries of the object. The rotation angle is calculated for the line corresponding to the longest, straight and continuous face of the object. Like the previous one, this method is suitable for normalizing the orientation of an object with direct boundaries. To isolate boundaries, one can use the Canny algorithm or the Sobel operator. Figure 3 shows the scheme of the preliminary processing algorithm with the described method.

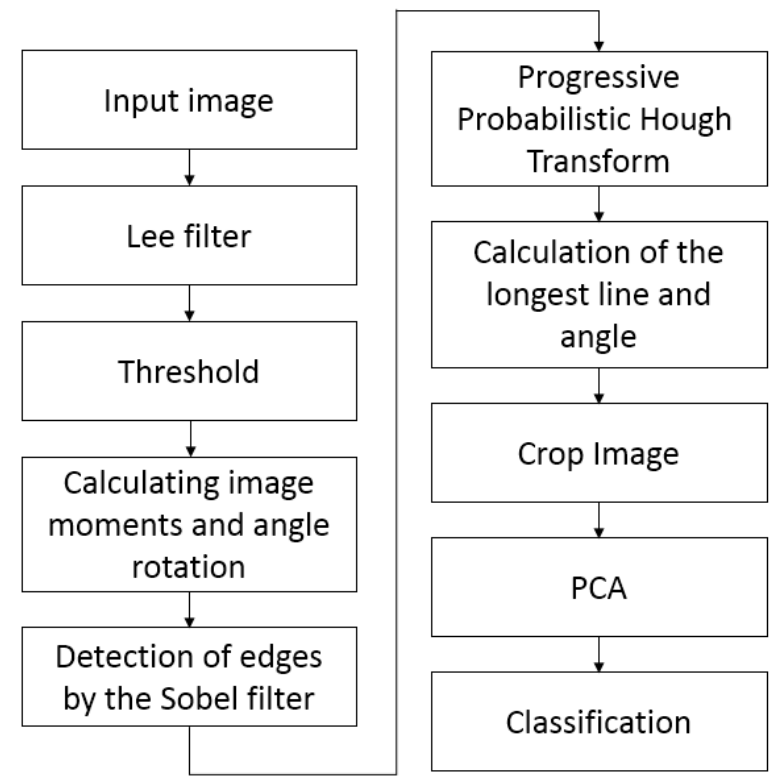

Figure 3. Preprocessing algorithm using the method based on finding the rectangle of the minimum area.

An example of this method is shown in Figure 4.

Table 1 shows the percentage of the number of images deviated from the true rotation angle by a specified angle to the total number of images.

The images obtained after normalization of the object are cut off. 

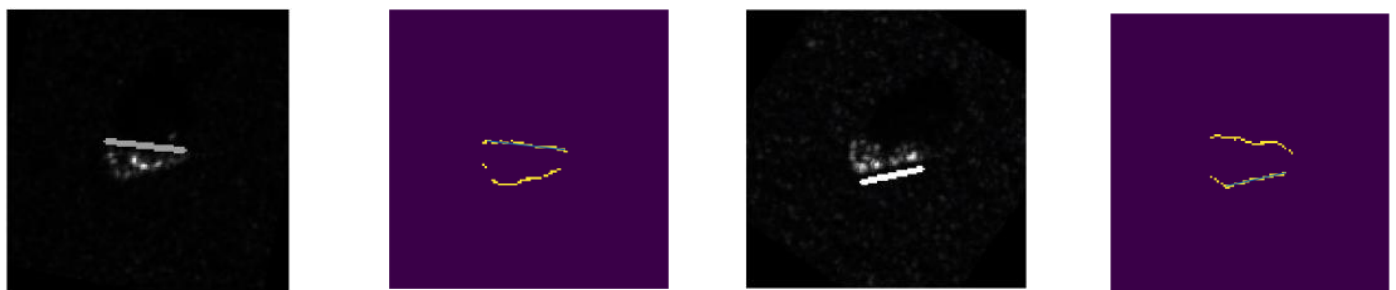

Figure 4. The first two images show an incorrect finding of the face of the object, the third and fourth demonstrate the correct operation of the method.

Table 1. Comparison of the results of the methods used to normalize the orientation of the object.

\begin{tabular}{llll}
\hline $\begin{array}{l}\text { Deviation from the true angle } \\
\text { of rotation }\end{array}$ & Image moments, \% & $\begin{array}{l}\text { Rectangle with min. } \\
\text { area, } \%\end{array}$ & Hough Transform, \% \\
\hline$>30$ & 4.276 & 9.922 & 6.079 \\
$20-30$ & 8.697 & 10.678 & 6.399 \\
$10-20$ & 37.056 & 25.778 & 9.862 \\
$5-10$ & 25.916 & 20.367 & 14.251 \\
$2-5$ & 14.049 & 18.621 & 30.163 \\
$1-2$ & 4.945 & 7.390 & 15.707 \\
$<1$ & 5.061 & 7.244 & 17.539 \\
\hline
\end{tabular}

\section{Principal component analysis}

Principal Component Analysis (PCA) is one of the most widely used methods for reducing the dimensionality of a feature space with the loss of the least amount of information. This method reduces to calculating the eigenvalues of the covariance matrix of the analyzed image. [2] Algorithms for calculating the covariance matrix operate in the line-by-line mode of reading the image, which allows achieving high performance and low requirements for the amount of RAM. [3]

\section{Classification algorithms}

\subsection{K-Neighbors}

K-nearest neighbors algorithm refers to metric classification algorithms with training sample $\Omega_{0}$. Such algorithms refer object $u$ to that class $y \in \mathrm{Y}$, for which the total weight of the nearest objects from the training sample is maximal:

$$
a\left(u, \Omega_{\mathrm{O}}\right)=\arg \max _{y \in Y} \Gamma_{y}\left(u, \Omega_{\mathrm{O}}\right), \text { и } \Gamma_{y}\left(u, \Omega_{\mathrm{O}}\right)=\sum_{i=1}^{\mathrm{K}}\left[y_{u}^{(i)}=y\right] \omega(i, u),
$$

where the weight function $\omega(i, u)$ estimates the degree of importance of the $i$-th neighbor for the classification of the object $u$. The function $\Gamma_{y}\left(u, \Omega_{0}\right)$ is an estimate of the closeness of the object $u$ to the class $y$. The importance function is chosen to be non-negative and not increasing in $i$. The selection criteria are due to the fact that the smaller the distance between the sampled objects $u$ and $x_{u}^{(i)}$, the greater the probability of a correct classification. In the algorithm $k$ of the nearest neighbors, the object $u$ is referred to a class with more elements among the $k$ nearest neighbors $x_{u}^{(i)}, i=\overline{1, k}$ :

$$
\omega(i, u)=[i \leq k] \omega_{i}, a\left(u, \Omega_{0}, k\right)=\arg \max _{y \in Y} \sum_{i=1}^{k}\left[y_{u}^{(i)}=y\right] \omega_{i} .
$$

The article used the Minkowski metric. It can be regarded as a generalization of the Euclidean and Manhattan distances. For the parameter $p=2$, the Minkowski distance is generalized to the Euclidean distance, and for $\mathrm{p}=\infty$ - to the Chebyshev distance. This metric is defined by the following formula:

$$
r(x, y)=\left(\sum_{i=1}^{n}\left|x_{i}-y_{i}\right|^{p}\right)^{1 / p} .
$$

The drawbacks of metric algorithms include storage of the entire training sample.

\subsection{Decision Tree (C4.5, CART)}

Decision Tree is a structure of a hierarchical type, in which branches a partition of the feature space is defined, and the sheets are elementary classification functions. There are various methods for constructing trees. In this paper, the algorithms C4.5 [4] and CART [5] are considered. 
C4.5 builds the source tree based on the input sample $\Omega_{\mathrm{O}}$. If all objects in the sample belong to the same class or the sample is small, then the tree is a sheet marked with the most common class in the sample. Otherwise, a split criterion is selected that divides the sample into two or more samples. Then the criterion is chosen for the obtained partitions. This procedure is recursively applied to each sample received. One of the criteria used, which is used in the work, is to minimize the entropy value of the obtained sample partitions. The resulting source tree is then trimmed to avoid retraining. Based on the received tree, a decision function is constructed for classifying objects.

In the CART algorithm, a binary decision tree is recursively constructed. The tree is created to the maximum size without using the stopping rule, and then it is clipped. The algorithm builds not one but a sequence of nested truncated trees. The best division is selected based on the sliding control. The partition criterion is based on the Gini index.

\subsection{SVM}

The support vector machine is one of the most reliable methods among all known algorithms and is most often used for comparison with new algorithms. The function separating the classes is a separating hyperplane. The algorithm maximizes the shortest distance between the points closest to the points on the hyperplane [6]. In this paper, the radial basis function is used as the separating function.

\subsection{AdaBoost}

A popular boosting algorithm AdaBoost was suggested by Freund and Schapire [7]. The main idea of the algorithm is to train a set of weak learners, such as small decision trees, on the prepared data. After this, the predictions from all of them are combined by weighted majority voting to obtain the final prediction. At the boosting iteration, the algorithm changes the weights for each sample from the training sample. Initially, the weighting factors are equal. For each subsequent iteration, the sample scales change individually. The weight of data that has been classified incorrectly is increasing. Thus, the algorithm concentrates training on the hard-to-class data.

\subsection{Random forest}

Random Forest is a set of decision trees. The decision to classify is made by voting the majority. Each decision tree is built independently of the others. For each tree, a subsample of the training sample is selected. For splitting the tree, the best attribute is selected. Typically, the tree is built before the exhaustion of the sample, the leaves of the tree should contain representatives of only one class.

\subsection{Convolutional Neural Network}

A convolutional neural network $(\mathrm{CNN})$ consists of one or more convolutional layers and subsequent one or more fully connected layers. $\mathrm{CNN}$ is often used to recognize objects, images, as well as to detect and segment objects. The use of GPUs made it possible to significantly accelerate the training of the neural network and to reduce the time for conducting experimental studies. Classification algorithms based on convolutional neural networks make it possible to achieve better results in image recognition and stability to rotate and shift the object in the image.

\subsection{Residual Neural Network}

Residual networks allow the construction of much deeper networks than conventional convolutional networks. The article [8] describes the use of an ensemble of six residual neural networks, each of which had up to 152 layers.

The residual neural network consists of residual blocks, including a pair of layers, where the input of the first layer is added to the output of the second layer. Figure 5 shows the residual block.

\section{Evaluation of classification results}

To assess the results of the classification, the sliding control method is used. Cross-validation is a statistical method for assessing the generalization of the quality of classification. It is a more reliable and thorough assessment method, compared to the usual sequential division of a data set into a 
training and test sample. With cross-validation, the data is repeatedly divided into training and test sets and fed to the classifier's input.

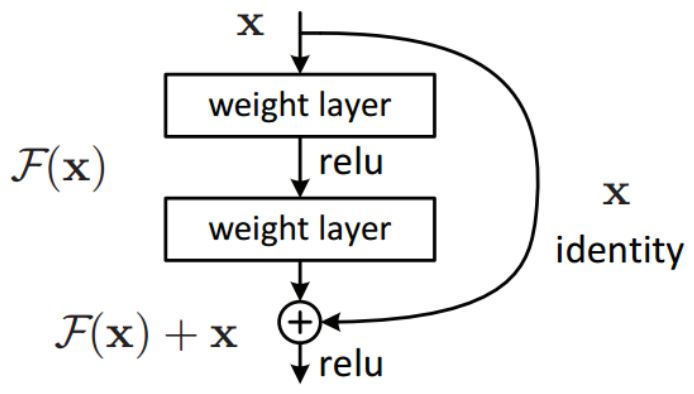

Figure 5. Residual block in the residual neural network.

The paper uses a modified method of sliding control with multiple partitioning [9] in which the entire volume of data is divided into a specified number of parts of $\mathrm{N}$ (equal to 1). The number of iterations of learning in this algorithm corresponds to the number of blocks $\mathrm{N}$. There is also a stratification of classes and samples, allowing to reduce the dispersion of estimates of sliding control. This leads to a decrease in the confidence interval and a more accurate classification quality. Applying class stratification makes it possible to break each class in a given ratio. At each iteration of the algorithm, K parts are randomly selected as the training sample and L parts as the test sample. This partition can be described as follows:

$$
\mathrm{N} \geq \mathrm{K}+\mathrm{L}, \Omega_{\mathrm{O}} \cup \Omega_{\mathrm{T}}=\Omega^{\prime}, \Omega^{\prime} \subseteq \Omega, \Omega_{\mathrm{O}} \cap \Omega_{\mathrm{T}}=\emptyset, \Omega_{\mathrm{O}}=\cup_{i=0}^{\mathrm{K}-1} \Omega_{\mathrm{O} i}, \Omega_{\mathrm{T}}=\cup_{j=0}^{\mathrm{L}-1} \Omega_{\mathrm{T} j}
$$

Where $\Omega_{\mathrm{O}}$ is a training sample, $\Omega_{\mathrm{T}}$ is a test sample, $\Omega$ is the original sample.

For each partition obtained, the classifier is set up on the training sample and the quality value of the classifier is calculated on the test sample.

The functional quality of the algorithm on the sample has the following form:

$$
\operatorname{CV}\left(\mu, \Omega^{\prime}\right)=\frac{1}{\mathrm{~N}} \sum_{p=1}^{\mathrm{N}} \frac{1}{\mathrm{~N}} \sum_{q=1}^{\mathrm{N}} \mathrm{Q}\left(\mu\left(\Omega^{\prime} \backslash \Omega_{\mathrm{O} p q}\right), \Omega_{\mathrm{O} p q}\right),
$$

where $\mu$ is the learning method.

\section{Experiments}

All the experimental studies were conducted on Intel Core i5-6600, 16 GB of RAM, NVIDIA GeForce GTX 970. All classification algorithms were written in Python 3.6. Also used were frameworks and libraries scikit-learn, scikit-image, openCV, numpy, tflearn, tensorflow. As objects of classification, samples of military equipment from the public database of radar images MSTAR.

For recognition, the magnitudes of the images of BMP-2, BTR-60, BTR-70 and T-72 were used. Prior to the classification, preliminary processing and cropping of images to a size of $60 \times 60$ pixels described in paragraph 3 was carried out. Classification by neural networks was carried out without preliminary image processing.

The target shooting angle is 15 and 17 degrees. The initial sample consists of 3438 images. For all images from the general sample, the dimension was reduced.

The value of the classification quality will be calculated as the average relative number of correctly classified objects from the test sample $\Omega_{\mathrm{T}}$. For the sliding control method, we specify the number of partitions and the number of iterations $\mathrm{N}=10, \mathrm{~K}=6$ and $\mathrm{L}=4$, dividing the total sample in the ratio $6: 4$. The method of the main components will reduce the dimensionality, retaining a significant part of the radar image information necessary for the classification of objects. The results of the classification, together with the results obtained in [12] and [13] are shown in Table 2.

The algorithms AdaBoost and Random forest significantly outperformed the results of CART and C4.5. Perhaps a further selection of parameters will help to achieve values that showed the method of nearest neighbors and the method of support vectors. Although the method based on Hough Transform showed the smallest average deviation of the obtained angle from a known value, the accuracy classification with this pre-treatment method gave way to the classification accuracy using the other two methods because of the high mean square deviation. 
Table 2. Results of classification.

\begin{tabular}{llllll}
\hline Classifier & $\begin{array}{l}\text { Image } \\
\text { moments }\end{array}$ & $\begin{array}{l}\text { Rectangle with } \\
\text { min. area }\end{array}$ & $\begin{array}{l}\text { Hough } \\
\text { Transform }\end{array}$ & $\begin{array}{l}\text { Known } \\
\text { rotation } \\
\text { angle }\end{array}$ & $\begin{array}{l}\text { Without } \\
\text { preprocessing }\end{array}$ \\
\hline KNeighbors & 0.93837 & 0.91904 & 0.89862 & 0.97689 & - \\
CART & 0.72500 & 0.74856 & 0.70908 & 0.82137 & - \\
C4.5 & 0.75218 & 0.75196 & 0.71672 & 0.84055 & - \\
SVM & 0.93263 & 0.93299 & 0.90887 & 0.97071 & - \\
AdaBoost & 0.90029 & 0.87232 & 0.87769 & 0.94365 & - \\
Random & 0.90320 & 0.89113 & 0.86926 & 0.94851 & - \\
forest & & & & & \\
CNN & - & - & - & - & 0.97516 \\
ResNet & - & - & - & - & 0.96535 \\
\hline
\end{tabular}

Classification algorithms based on the use of convolutional neural networks have surpassed the other classification algorithms studied in the case of methods of object orientation normalization. The method of nearest neighbors and the method of support vectors for a known angle of rotation of the target show a similar classification accuracy. Due to the fact that in practical classification tasks the angle of object rotation is rarely known, the application of classification algorithms based on convolutional neural networks will allow achieving better results even without preliminary image processing.

\section{Conclusion}

In this paper, we compare the classification algorithms in the problem of object recognition on radar images. Various methods of preliminary image processing and object orientation normalization were presented and studied. The experimental studies carried out have shown the dependence of the classification accuracy on the value of the root-mean-square deviation of the image rotation angle. It is shown that the application of classification algorithms based on convolutional neural networks allows obtaining the best classification accuracy even without preliminary image processing.

A further area of research is the development of a classification algorithm based on a convolutional neural network and the selection of the best hyperparameters, which allows to exceed the obtained classification accuracy.

\section{References}

[1] Ross T, Worrel S, Velten V, Mossing J and Bryant M 1991 Standard SAR ATR evaluation experiments using the MSTAR public release data set Proc. SPIE - The International Society for Optical Engineering 3370 566-573

[2] Turk M 1998 Pentland, Eigenfaces for recognition J. Cognit. Neurosci 3(1) 71-86

[3] Kuznetsov A V and V V Myasnikov 2014 A comparison of algorithms for supervised classification using hyperspectral data Computer Optics 38(3) 494-502

[4] Salzberg S L 1994 C4.5: Programs for Machine Learning Machine Learning 16(3) 235-240

[5] Wu X 2008 Top 10 algorithms in data mining Knowledge and Information Systems 14(1) 1-37

[6] Cortes C and Vapnik V 1995 Support-Vector Networks Machine Learning 20(3) 273-297

[7] Freund Y and Schapire R E 1997 A Decision-Theoretic Generalization of On-Line Learning and an Application to Boosting Journal of Computer and System Sciences 55(1) 119-139

[8] He K, Zhang X, Ren S and Sun J 2016 Deep residual learning for image recognition Proceedings of the IEEE Computer Society Conference on Computer Vision and Pattern Recognition 2016 770-778

[9] Vorontsov K V 2004 Combinatorial substantiation of learning algorithms Computational Mathematics and Mathematical Physics 44(11) 1997-2009

[10] Zherdev D A, Minaev E Y, Procudin V V and Fursov V A 2017 Object recognition using real and modelled SAR images Procedia Engineering 201 503-510

[11] Zherdev D A, Kazanskiy N L and Fursov V A 2014 Object recognition by the radar signatures 
of electromagnetic field scattering on base of support subspaces method Computer Optics 38(3) 503-510

[12] Borodinov A A and Myasnikov V V 2018 Comparison of classification algorithms for various methods of preprocessing radar images of the MSTAR base Proceedings of SPIE 10696 1069614-7

[13] Borodinov A A and Myasnikov V V 2017 Comparison of classification algorithms in the task of object recognition on radar images of the MSTAR base CEUR Workshop Proceedings 190137 41

\section{Acknowledgments}

This work was supported by the Russian Foundation for Basic Research (RFBR) grant 17-29-03190, 18-01-00748 A and by the Federal Agency of scientific organization (Agreement 007-GZ/43363/26). 